\title{
Introduction to this special issue on statistics for wildfire processes
}

\author{
Marcia Gumpertz
}

This special issue on statistics for wildfire processes brings together foresters, wildfire ecologists, statisticians, mathematicians, and economists. All of these disciplines bring different interests, approaches and expertise to the modeling of wildfire processes. It is not necessarily easy, however, to communicate across disciplines or follow the developments in a variety of fields. Hence, the papers in this issue are primarily expository in nature, with the goal of facilitating and cross-fertilizing cross-disciplinary research on wildfire modeling. The papers in this issue were first presented in two conferences that brought wildfire modelers together. The first seed of the idea for the special issue was planted in a theme session of the 2005 annual meeting of the Eastern North American Region (ENAR) of the Biometric Society in Austin, TX, titled "Statistical Analysis of Wildfire Data." The project was fertilized and grew in May of that year at a 5-day "Workshop on Forest Fires and Point Processes" at the Fields Institute in Toronto.

This issue begins with a paper by B. M. Wotton explaining the Canadian forest fire danger rating system, which is used by Canadian fire management agencies. Fire managers use models to help predict forest fire growth and intensity and make decisions each day about where to position fire fighting resources. This paper gives statistical modelers a view of these uses of fire models, the current types of models employed by fire managers, and the need for understanding how the relationship between the Canadian Fire Weather Index System outputs and fuel moisture and the probability of fire occurrence vary among regions of the country and forest types.

The next two papers describe ideas for stochastic wildfire models that could potentially be used for forecasting the spread of fires that have already started. The first of these, by Boychuk et al., describes a model in which fire spreads from cell to

M. Gumpertz (区)

Office for Diversity and African American Affairs, North Carolina State University, Raleigh, NC, USA

e-mail: gumpertz@ncsu.edu 
cell. The model is a continuous-time Markov chain on a lattice, and is an example of an interacting particle system. This type of model allows predictions of the growth of a wildfire, including different courses that a fire could take and construction of probability contours of area burned. Ivanoff proposes the use of renewal processes for modeling a crown fire which is spread by a prevailing wind. She provides a lucid exposition of multi-parameter renewal processes. This type of model is not in current use for modeling wildfire spread, but has attractive features and is proposed as a jumping-off point for developing more realistic models.

Four papers on different kinds of point processes for modeling the distribution of fires in space and time follow. First Vere-Jones gives background on space-time point process models in which the onset times of events are the time components of the points and the size or intensity of the event are marks associated with the events. This paper assumes previous familiarity with point processes and focuses on the special features of space-time processes. Vere-Jones then shows some specific point process models that have been developed for seismology and discusses some similarities and differences between earthquakes and wildfire processes. The next paper, by R. Turner, demonstrates the fitting of non-homogeneous spatial point process models to wildfire data from New Brunswick, Canada, from 1987 through 2003. The approach is to fit the long distance spatial trend with nonparametric smoothing and the remaining spatial dependence among fire locations with an interaction model. This paper gives a detailed tour of practical issues such as cleaning and organizing the data, software to fit the models, dangerous pitfalls to avoid, judgment calls that must be made, and evaluating the fit of the model. The paper by Hering et al. uses similar non-homogeneous spatial point processes to model the spatial distribution of wildfires in the St. Johns River Water Management District in Florida. This paper provides an example and a simulation demonstrating the behavior of the homogeneous $K$-function for detecting complete spatial randomness when spatial trend is present but not recognized. Hering et al. also discuss $K$-cross functions for studying the relationship between two processes when spatial trend is present. Schoenberg et al. focus on developing models to predict the probability of an ignition at a given location under given weather conditions. They use kernel smoothing to estimate the effects of meteorological variables on the intensity parameter of a separable point process model, and then compare the resulting model to one based on the commonly used burning index (BI) for forecasting wildfire incidence in Los Angeles County.

The next paper in this special issue discusses the effects of sampling density and censoring of data on wildfire modeling. This paper by Moritz et al. gives background on how wildfire data are collected and a brief introduction to fire ecology. It then provides an exposition of survival analysis for the study of fire frequency. The final paper of the special issue, by D. Butry, discusses the difficulty of inferring causality in models of wildfire data. The question at issue is whether prescribed burning or wildfire suppression efforts actually reduce the number of acres burned compared to how much would have burned if no intervention had been undertaken. Butry applies propensity score matching methods, used in labor economics to evaluate the impact of social programs, to the problem of evaluating the impact of prescribed burning on wildfire size. 